\title{
Research on Construction of Enterprise Informatization based on Model of Multi-agent RigidFormation
}

\author{
Li Sun ${ }^{1, \mathrm{a}}$, Ying $\mathrm{Liu}^{1, \mathrm{~b}}$, Qiang $\mathrm{Qu}^{2, \mathrm{c}}$, Lilin $\mathrm{Ma}^{2, \mathrm{~d}}$, Guang Yang ${ }^{1, \mathrm{e}}$ and $\mathrm{Hu} \mathrm{Cao}^{2, \mathrm{f}}$ \\ ${ }^{1}$ CNGC Harbin First Machinery Group Co., Ltd., Harbin, \\ Heilongjiang Province, P.R. China \\ ${ }^{2}$ CNGC China North Advanced Technology Generalization Institute, \\ Beijing City, P.R. China

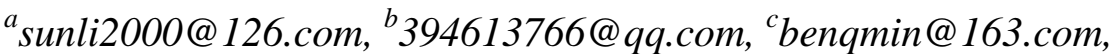 \\ dmall329@163.com, ${ }^{e} 1103856428 @ q q . c o m,{ }^{f}$ job_caohu@163.com
}

\begin{abstract}
Enterprise informatization is a large system including: input, output, feedback, operation inside, executive action and incentive measures. Inner-enterprise can be seemed as an artificially social organization unit composing of various agents in a rigid formation. This paper mainly proposes an enterprise informatization associated with rigid formation theory of multi-agent. The connection between agents and enterprise factors is represented. The relationship between enterprise factors is described by potential function theory: the attractive force presents that all factors have the same opinion, and the repulsive force presents that all factors have the different opinion needed to be solved. Finally, the simulation proves that the model mentioned in this paper is effective.
\end{abstract}

Keywords: informatization; multi-agent; rigid; potential function

\section{Introduction}

Nowadays, the world is transforming from the traditional industry society to the modern informatization one. "Using informatization to promote industrialization, using industrialization to develop informatization, and help fuse informatization and industrialization together" has become the economic development of the important strategic deployment in China. At present, informatization is already accepted by various enterprises. The enterprises hope to develop from the overall consideration of information management, and establish an effective enterprise information management platform and the management system. However, because of the later start and the weaker basis, the enterprises of China are badly in need of related policy and theoretical guidance. As a result, theory and method of enterprise information construction research have very important practical significance and application value.

At present, multi-agent is very popular and is attracting many domestic and foreign scholars' research interests [1-7]. In the study of multi-agent theory and enterprise information, for examples of government information, $\mathrm{Wu}$ [8] proposes the causality diagram system theory for thinking swarm behavior of the government information, and use the multi-agent method to establish the government informatization EGGBM of behavior model; Li [9] proposes a new urban traffic control system based on multi-agent system framework, in view of the existing traffic control system of weakness on low efficiency deficiency, slow real-time reactivity and so on. This paper gives an intensive study on enterprise informatization based on formation control of multi-agent, and important conclusions are supplied in the end. 


\section{Preliminaries}

\subsection{Graph Rigidity}

To introduce graph rigidity we define $G=(V, E)$ to be an undirected complete graph with $\mathrm{n}$ vertices. Define the composite vector $p=\left(p_{1}, p_{2}, \ldots p_{n}\right) \in R^{2 n}$ and a pair $(G, p)$.

Denotethe constant parameter $d_{i j}=\left\|p_{i}-p_{j}\right\|$ as the Euclidean distances between pairs of points $\left(p_{i}, p_{j}\right)$. In addition, the introduction of rigidity matrix in [10] claims that:

$$
\left\langle\left(p_{i}-p_{j}\right),\left(p_{i}-p_{j}\right)\right\rangle=d_{i j}^{2} \quad i, j \in\{1,2, \ldots, n\}
$$

Assumethat the trajectory is smooth, then we could get the following expressions :

$$
\begin{gathered}
2\left\langle\left(p_{i}-p_{j}\right),\left(p_{i}-p_{j}\right)\right\rangle=0, \\
i, j \in\{1,2, \ldots, n\}, t \geq 0
\end{gathered}
$$

where $p_{i}$ is the velocity at point $p_{i}$. Then, we obtain the following homogeneous equation by combining (2) at different points:

$$
R \stackrel{\square}{p=0}
$$

where $p=$ column $\left\{p_{1}, p_{2}, \ldots p_{n}\right\}$, and $R$ is called rigidity matrix with dimension $m \times 2 n$ $[m=n(n-1) / 2]$ in the plane.

In addition, the rigidity function is describedas follows for another way of the definition of rigidity matrix:

$$
\hat{g}_{G}(p)=\left[\left\|p_{e_{1}}^{\text {in }}-p_{e_{1}}^{\text {out }}\right\|^{2},\left\|p_{e_{2}}^{\text {in }}-p_{e_{2}}^{\text {out }}\right\|^{2}, \ldots\left\|p_{e_{m}}^{\text {in }}-p_{e_{m}}^{\text {out }}\right\|^{2}\right]^{T}
$$

where the $i$ th component $\left\|p_{e_{k}}^{\text {in }}-p_{e_{k}}^{\text {out }}\right\|^{2}$ corresponds to the edge $e_{k}$ of $E(k \in\{1,2, \ldots, m\}$ $\left.E=\left\{e_{1}, e_{2}, \cdots e_{m}\right\}\right)$.

Also we define the rigidity function associated with the framework $(G, p)$ as the function $g_{G}: R^{2 n} \rightarrow R^{|E|}$

$$
g_{G}:=\left(\cdots,\left\|p_{k}-p_{j}\right\|^{2}, \cdots\right)
$$

The $i$ th component $\left\|p_{k}-p_{j}\right\|^{2}$ corresponds to the edge $e_{i}$ of $E$.

There are three similar definitions of rigidity in graph: rigidity, global rigidity, and infinitesimal rigidity $[11,12]$ :

Definition 2.1 A framework $(G, p)$ is rigid if there exists a neighborhood $U \subset R^{2 n}$ of $\mathrm{p}$ such that:

$$
g_{G}^{-1}\left(g_{G}(p)\right) \cap U=g_{K}^{-1}\left(g_{K}(p)\right) \cap U
$$

where $\mathrm{K}$ is the complete graph with the same vertices as $\mathrm{G}$.

Definition 2.2 A framework $(G, p)$ is globally rigid if $g_{G}^{-1}\left(g_{G}(p)\right)=g_{K}^{-1}\left(g_{K}(p)\right)$

Definition 2.3 A framework $(G, p)$ is infinitesimally rigid in the plane if $\operatorname{dim}\left(\operatorname{ker} J_{g_{G}}(p)\right)=3$, or if

$$
\operatorname{rank} J_{g_{G}}(p)=2 n-3
$$


where $J_{g_{G}}(p)$ is rigidity matrix and it will be explained in part B.

Definition 2.4 Point $p$ are regular points of the graph $\mathrm{G}$ with $\mathrm{n}$ vertices if

$$
\operatorname{rank} J_{g_{G}}(p)=\max \left\{\operatorname{rank} J_{g_{G}}(q) \mid q \in R^{2 n}\right\}
$$

Definition 2.5Point $p$ is a regular point of the graph $G$ with $n$ vertices if

$$
\operatorname{rank} J_{\hat{g}_{G}}(p)=\max \left\{\operatorname{rank} J_{\hat{g}_{G}}(q) \mid q \in R^{2 n}\right\}
$$

From Definition 2.4, we know the following theorem:

Theorem 2.1[12]A framework $(G, p)$ is infinitesimally rigid if and only if $(G, p)$ is rigid and $p$ are regular points.

Theorem 2.1 tells us that some framework is rigid but not infinitesimally rigid. However, if the framework is infinitesimally rigid, then it is sure to be rigid. Figure 1 illustrates these properties with two examples. It is easily to compute that rank $J_{g_{G}}(q)=2 n-3$ in Figure 1 (a) and $\operatorname{rank} J_{g_{G}}(q)<2 n-3$ in Figure 1 (b), so Figure 1 (a) is rigid and infinitesimally rigid; Figure 1 (b) is rigid but not infinitesimally rigid, as $p$ are not regular points. In general, the rigid but fail to be infinitesimally rigid graph almost have parallel or collinear edges. In this paper, rigid means infinitesimally rigid.

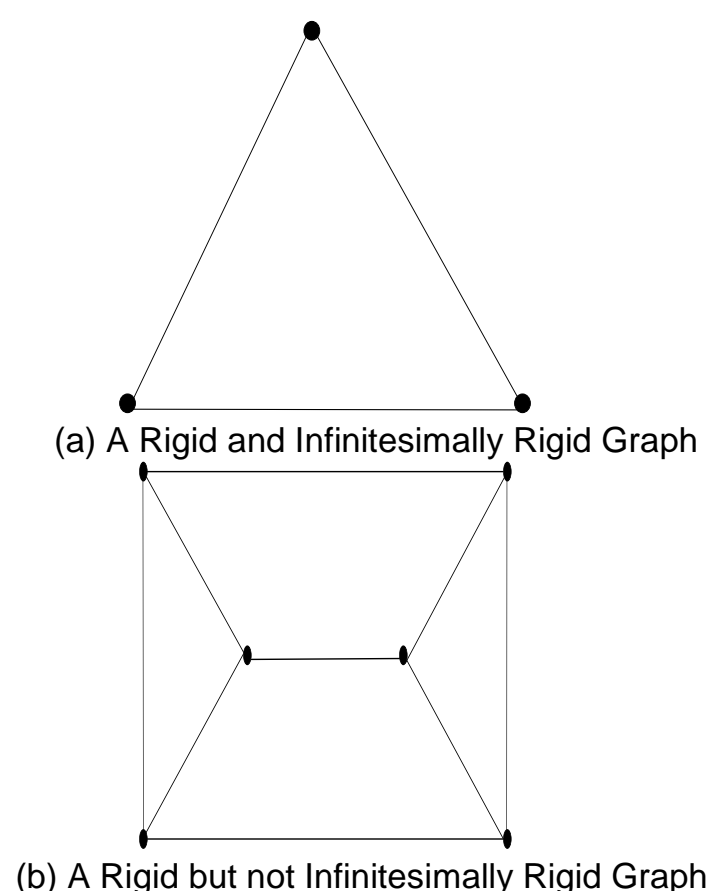

Figure 1. The Two Possible Examples with Rigid Framework

Lemma 2.1[13] Consider a formation $F$ in $R^{2}$, with agents in generic positions and with defined agent pairs having the inter-agent distances maintained. Let $G=(V, E)$ be the undirected graph. Then $F$ is rigid if and only if there is a subset $E^{\prime} \subseteq E$ satisfying the following two conditions:

(1) $\left|E^{\prime}\right|=2|V|-3$.

(2) For all $E^{\prime \prime} \subseteq E^{\prime}, E^{\prime \prime} \neq \emptyset$, 
$\left|E^{\prime \prime}\right| \ll 2\left|V\left(E^{\prime \prime}\right)\right|-3$, where $\left|V\left(E^{\prime \prime}\right)\right|$ is the number of vertices which are the end vertices of the edges in $E^{\prime \prime}$.

\subsection{Rigidity Matrix}

Based on Part 2.1, in framework $(G, p)$ we define $d_{i j}=\left\|p_{i}-p_{j}\right\|$ the Euclidean distances between pairs of points $\left(p_{i}, p_{j}\right)$ and they are constant. Also, we can get [11]:

$$
\left(p_{i}-p_{j}\right) \square\left(p_{i}-p_{j}\right)=d_{i j}^{2} \quad i, j \in\{1,2, \ldots, n\}
$$

Assuming a smooth trajectory, XX can be differentiated:

$$
2\left(p_{i}-p_{j}\right) \square\left(p_{i}-p_{j}\right)=0, \quad i, j \in\{1,2, \ldots, n\}, t \geq 0
$$

where $p_{i}$ is the velocity of point $p_{i}$, and we collect (5) into a new equation:

$$
J_{g_{G}}(p) p=0
$$

where $J_{g_{G}}(p) p=0, p=\operatorname{column}\left\{p_{1}, p_{2}, \ldots p_{n}\right\}$, and $J_{g_{G}}(p)$ is the rigidity matrix with structure $m \times n d$ where $m=C_{n}^{2}$.

\section{Gradient Control Laws for Multi-Agent Rigid System}

It is well known that the collective objective of flocking motion can be described by the relative positions and relative velocities of the agents. Hence the desired relative positions are uniquely determined by utilizing the flocking vector $f=\left(f_{1}, f_{2}, \ldots, f_{N}\right)^{T} \in R^{m N}, f_{i} \in R^{m}, \forall i$. Define the goal topology of a multi-agent system as $G_{g}=\left(V_{g}, E_{g}\right)$, the edge set is given by:

$$
E_{g}=\left\{\left(n_{i}, n_{j}\right)\left\|\mid f_{i}-f_{j}\right\|=d_{i j}<R_{d}, n_{i}, n_{j} \in V_{g}, i \neq j\right\}
$$

where $R_{d}$ is the communication radius of agents.

The definition of stable infinitesimally rigid formation has given a flocking vector $f=\left(f_{1}, f_{2}, \ldots, f_{N}\right)^{T}$ and assume that $G_{g}=\left(V_{g}, E_{g}\right)$ is connected. Then a multi-agent system is called a stable infinitesimally rigid iff for any $\left(n_{i}, n_{j}\right) \in E_{g}$, it satisfies:

$$
\left\{\begin{array}{l}
x_{i}-x_{j}=f_{i}-f_{j}=d_{i j} \\
v_{i}=v_{j} \\
\operatorname{rank} J_{g_{G}}(f)=2 n-3
\end{array}\right.
$$

On the whole, the main idea of the proposed flocking control strategy is to relate the desired rigid geometricconfigurations of the goal topology to the local or global extremes of the group potential functions [14]. The distributed control law $u_{i}$ is designed by using the state information from agent $i$ and its neighbors hence can be described in the following form:

$$
u_{i}=\underbrace{-\sum_{j \in N_{i}\left(G_{c}\right)} \nabla_{x_{i j}} V_{i j}\left(\left\|x_{i j}\right\|\right)}_{u_{i}^{1}}-\underbrace{\sum_{j \in N_{i}\left(G_{c}\right)} a_{i j}(t)\left(v_{i}-v_{j}\right)}_{u_{i}^{2}}
$$


Where $u_{i}^{1}$ acts as the induced term for achieving the desired rigid configuration, $u_{i}^{2}$ is the velocity consensus term and is responsible for aligning the agent velocities to a common value. $V_{i j}$ represents the artificial potential function between agents $i$ and $j$.

Under the assumption that the communication topology is connected while the robots are in the radius of communication during the whole process of evolution, it can be proved that the whole group will asymptotically achieve the desired stable rigid motion. However, in practical situations, Since it is required that all the agents attain a common velocity while maintaining the desired group shape, it is desired that $v_{i j}(t) \rightarrow 0$ and $x_{i j}(t) \rightarrow f_{i j} \forall i, j \in V$, where $f_{i j}$ is the desired distance between agents $i$ and $j$ in the goal topology. Furthermore, it is assumed that $f_{i j}$ is also compatible in the sensethat $f_{i k}+f_{k j}=f_{i j}, \forall i, j, k \in V$.

Then the rigid flocking potential function $V_{i j}$ should be devised for the edges in $E_{i j}(t) \cap E_{g}$ based on the following principles.

\section{Enterprise Informatization and Multi-Agent Rigid System}

Enterprise informationization refers to the enterprises to select the advanced computer communication network, other modern technology and equipment, the production and marketing, sales management research, development management, policy-making, the construction of network and application system, fully develop extensive use of enterprise internal and external information resources, adjust or reconstruct enterprise organization structure and business model, decision system for the enterprise to provide accurate and effective data information. The goal is to build the ability of rapid response to the market and enhance the core competitiveness of the enterprise.

Multi-agent rigid system includes lots of agents, and it has obvious advantages compared to a single agent system:

The multi-agent coordination may help do complex works which can't be completed by a single agent; for the same task, single agent system has more design and manufacturing difficulty than simple agent; multi-agent coordination can largely improve the robustness and fault tolerance of the whole system.

From the perspective of physical significance, multi-agent rigid system is not an algebraic addition of some purely single simple agents, but its effect includes linear summation of single agents and the increment of agents' interaction. The interaction between the individual consists of two factors: coordination and cooperation. Multi-agent coordination and cooperation control methods can be divided into two categories: centralized and distributed. Early planning method is mainly composed of centralized; but now, distributed planning method is focused by more and more scholars.

In centralized planning method, multi-agent rigid system could be seemed as a complicated agent which has various freedoms, and all the coordination control is completed by a unified planning in the system. The characteristics of centralized planning method are: it can obtain the optimal solution without considering the complexity of computing programming problem, but otherwise, centralized planning method needs much more time to execute the algorithm, so this method has worse timeliness.

In distributed planning method, each agent can independently collect information from their surroundings and neighbor agent movement information, and plan out a point of relative optimal path according to the collected information and its own state information to target. The characteristics of distributed planning method are: the distributed method can obtain high computing efficiency and running speed without considering planning 
problem about the condition of global optimal solution. However, on the condition that communication traffic increased significantly between neighbors, the computing time of distributed algorithm is probably equal or exceed centralized one, even not receive the optimal solve. In recent years, research of distributed programming method has made considerable development, and gradually becomes the mainstream of multi-agent coordination control method.

This paper mainly studies the enterprise informatization model based on distributed planning method; each agent is equivalent to an element of enterprise. A complete enterprise information process basically has the following several elements: Assets Operation Management System, OA system, Human Resource Management System, Quality Management System, Financial Management System, Logistics Management System, Production Management System. Each element can independently collect information about the surrounding environment and the neighbor's running status information, and adjust their behavior to adapt to the dynamic change of the surrounding environment. As this starting point, multi-agent formation model theory may successfully extend to the enterprise information construction theory.

\section{Model of Multi-Agent Informatization}

In the model of multi-agent informatization, the various agents mean the different informatization factors, and the relationship between them is as follows:

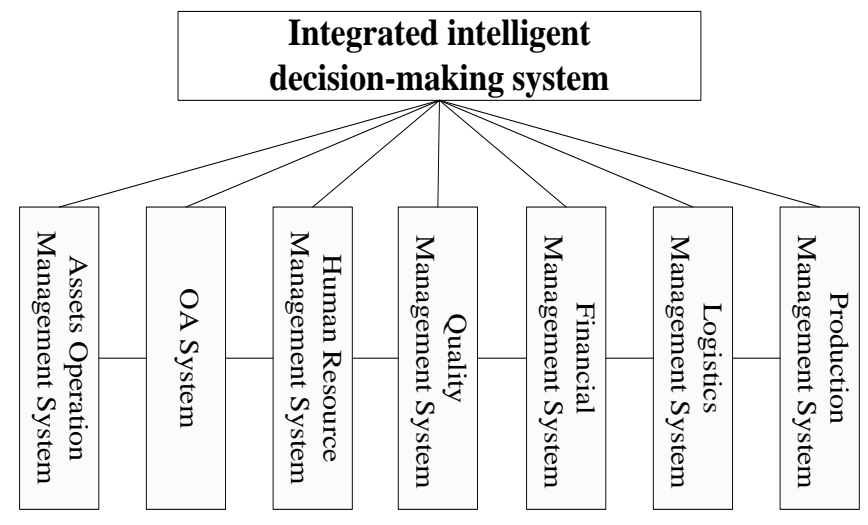

Figure 2. InformatizationModel of Multi-Agent

Enterprise informatization is the process of each agent from disordered state transferringto ordered and keeping running, the various informatization factors can be kept in a stable rigid state. Here we can learn from theory of articles ${ }^{[1,2]}$ using potential functions between agents in rigid formations. The potential functions produce a repulsive force between two intelligent agentswhen distance is less than the specified threshold, andgenerating attractive force when distance is more than the specified threshold, until finally reaching a stable operating state. From the perspective of enterprise information, it is thatthrough constraint rules (potential function), all departments and systems reach a stable operating condition.

To some multi-agent informatization model, the following smooth potential function is adopted: 


$$
\left.V_{i j}\left\|x_{i j}\right\|\right)=\left\{\begin{array}{l}
a \ln \left\|x_{i j}\right\|^{2}+\frac{b}{\left\|x_{i j}\right\|^{2}} \quad 0<\left\|x_{i j}\right\| \leq \sqrt{\frac{b}{a}} \\
a \ln \left\|x_{i j}\right\|^{2}+\frac{b}{\left\|x_{i j}\right\|^{2}}+\cos \left(1+\frac{\left\|x_{i j}\right\|^{2}-\frac{b}{a}}{R^{2}-\frac{b}{a}}\right) \pi+1 \quad \sqrt{\frac{b}{a}}<\left\|x_{i j}\right\|<R_{d} \\
a \ln \left\|x_{i j}\right\|^{2}+\frac{b}{\left\|x_{i j}\right\|^{2}}+2 \quad \text { otherwise }
\end{array}\right.
$$

where $V_{i j}$ is always nonnegative and differentiable in $\left(0, R_{d}\right)$, and $V_{i j}$ attains its unique global minimum when $\left\|x_{i j}\right\|$ obtains a predefined desired distance.

Also, in this equation, $a, b, R_{d}$ are all positive constant, and $b>\frac{e}{a}, R_{d}>\sqrt{\frac{b}{a}}, \quad V_{i j}$ means the potential function between agent $i$ and agent $j, \quad x_{i j}$ represents the distance between agent $i$ and agent $j$. From Figure 3 below, we know that $V_{i j}$ is continuously differentiable everywhere.

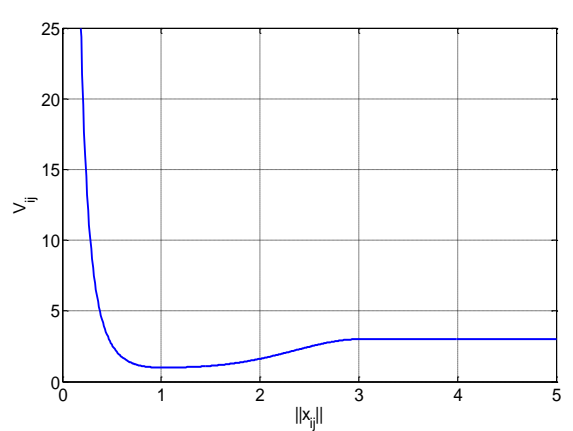

Figure 3. The Potential Function which is Continuously Differentiable Everywhere

The potential function $V_{i}$ of agent $i$ is as follows from the characterof $V_{i j}$.

$$
V_{i}=\sum_{j \notin N_{i}} V_{i j}(R)+\sum_{j \in N_{i}} V_{i j}\left(\left\|x_{i j}\right\|\right)
$$

Furthermore, the control law $u_{i}$ of agent $i$ is:

$$
u_{i}=-\sum_{j \in N_{i}} \nabla_{x_{i}} V_{i j}-\sum_{j \in N_{i}} w_{i j}\left(v_{i}-v_{j}\right)
$$

Where $w_{i j}$ is the weight coefficient of each edge, and it is responding to contacts tightnessbetween elementsin the model of multi-agent informatization.

\section{Experiments}

This section mainly utilizes robots simulation to demonstrate that the feasibility of multi-agent information model based on the rigid formation, considering the combined 
potential theory presented in this paper.The simulation is with five robots example, and eight-agent model also can be done in the same manner.

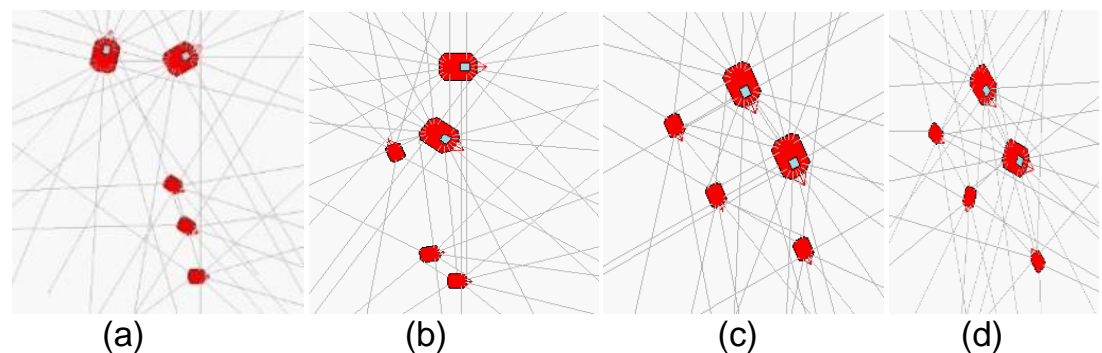

Figure 4. Experiments with Robots

In Figure 4, picture (a) shows that the robots are in the disordered state, and picture (b), (c), (d) describe that all robots (elements of enterprise) are reaching a ordered state by the potential function, and it also means that some constraint rules make elements of enterprise stabilized.

\section{Conclusions}

The paper mainly studies the model of enterprise informatization with multi-agent rigid formation theory, and claims that the relationship with agents and enterprise elements. The potential function is introduced to describe the connection of various enterprise elements: attractive force means that all elements supply the same opinions and repulsive force expresses that all elements have different ideas which are needed to be adjusted. The control law based on smooth potential function theory is also mentioned. Finally, the simulation between some robots is provided to prove that the theory and methods in this paper are innovative and effective.

\section{References}

[1] H. Cao, Y. Bai and H. Liu, "Distributed rigid formation control algorithm for multi-agent systems", Kybernetes, vol. 41, no. 10, (2012), pp. 1650-1661.

[2] H. Cao, Y. Bai, J. Chen and H. Fang, "Control of 2D minimally persistent formations with three coleaders in a cycle", International Journal of Advanced Robotic Systems, vol. 10, no. 21, (2013), pp. 1-7.

[3] C. Renolds, "Flocks, herds and schools: a distributed behavioral model", Computer Graphics, vol. 21, no. 4 , (1987), pp. 25-34.

[4] R. Olfati-Saber and R. Murray, "Distributed cooperative control of multiple vehicle formations using structural potential functions", Proceedings of 15th IFAC World Congress, (2002).

[5] V. Tran and S. Lee, "A stable formation control using approximation of translational and angular accelerations", International Journal of Advanced Robotic Systems, vol. 8, no. 1, (2011), pp. 65-75.

[6] H. G. Tanner, A. Jadbabaie and G. J. Pappas, "Stable flocking of mobile agents, part I: fixed topology", Proceedings of 42nd IEEE Conference on Decision and Control, (2003).

[7] H. G. Tanner, A. Jadbabaie and G. J. Pappas, "Stable flocking of mobile agents part II: dynamic topology", Proceedings of 42nd IEEE Conference on Decision and Control, (2003).

[8] J. Wu and B. Hu, "Multi-agent simulation for interaction of IT implementation and group behavior", Journal of Systems Engineering, vol. 24, no. 2, (2009), pp. 218-225.

[9] S. Li, "Application of MAS in Intelligent Transportation Systems", Urban Transport of China, vol. 4, no. 5, (2009), pp. 78-80.

[10] T. Eren, B. D. O. Anderson and A. S. Morse, "Operations on rigid formations of autonomous agents", Communications in Information and Systems, vol. 4, no. 3, (2004), pp. 209-219.

[11] L. Krick, M. E. Broucke and B. A. Francis, "Stabilization of infinitesimally rigid foramtions of multirobot networks", Proc. of the 47th IEEE Conference on Decision and Control, (2008); Cancun, Mexico.

[12] L. Asimow and B. Roth, "Rigidity of graphs", Transactions of the American Mathematical Society, vol. 252, (1978), pp. 279-289.

[13] G. Laman, "On graphs and the rigidity of plane skeletal structures", Journal of Engineering Mathematics, vol. 4, (1970), pp. 331-340.

[14] F. E. Schneider and D. Wildermuth, "Experimental comparison of a directed and a nondirected potential field approach to formation navigation", Proceedings of IEEE International Symposium on Computational Intelligence in Robotics and Automation, Espoo, Finland, (2005). 